\title{
Identifying Key Stakeholders as Part of Requirements Elicitation in Software Ecosystems
}

\author{
Stephanie Lewellen \\ Maynooth University \\ Maynooth, Co Kildare, Ireland \\ stephanie.lewellen.2020@mumail.ie
}

\begin{abstract}
Software ecosystems (SECOs) involve a number of actors that work together for a shared market. The software products within the software ecosystem typically have a common technological platform, and consist of a keystone player at the center of the ecosystem with niche players addressing market segments the keystone player would otherwise not have access to.

Stakeholder identification is critical to the financial and functional success of software development projects, however the task of identifying all stakeholders in a SECO is often not possible due to the high volume of stakeholders and open interfaces. The identification of key stakeholders should ensure that the most relevant requirements are elicited during a software planning cycle.

The objective of this research is to examine how key stakeholders can be identified in complex SECOs. This research takes a design science approach and the main component of the current research is the design of an artifact in the form of a reference process model, that is applied in a real-world environment to develop a business process model. Consequently, this research aims to facilitate academia and industry in SECO optimization, especially from a requirements management perspective.
\end{abstract}

\section{CCS CONCEPTS}

- Software and its engineering $\rightarrow$ Software product lines; • Applied computing $\rightarrow$ Cross-organizational business processes.

\section{KEYWORDS}

stakeholder identification, software ecosystems, software product lines, requirements elicitation

\section{ACM Reference Format:}

Stephanie Lewellen. 2020. Identifying Key Stakeholders as Part of Requirements Elicitation in Software Ecosystems. In 24th ACM International Systems and Software Product Line Conference - Volume B (SPLC '20), October 1923, 2020, MONTREAL, QC, Canada. ACM, New York, NY, USA, 8 pages. https://doi.org/10.1145/3382026.3431249

Permission to make digital or hard copies of all or part of this work for personal or classroom use is granted without fee provided that copies are not made or distributed for profit or commercial advantage and that copies bear this notice and the full citation on the first page. Copyrights for components of this work owned by others than the author(s) must be honored. Abstracting with credit is permitted. To copy otherwise, or republish, to post on servers or to redistribute to lists, requires prior specific permission and/or a fee. Request permissions from permissions@acm.org.

SPLC '20, October 19-23, 2020, MONTREAL, QC, Canada

(C) 2020 Copyright held by the owner/author(s). Publication rights licensed to ACM. ACM ISBN 978-1-4503-7570-2/20/10 . \$ \$15.00

https://doi.org/10.1145/3382026.3431249

\section{INTRODUCTION AND MOTIVATION}

Software companies are increasingly adopting techniques of reuse, variability, and partnering to reduce the cost of development and testing, and to be competitive in new market segments.

One option is a Software Product Line (SPL) approach, where a set of similiar software products share a number of common features but contain variation points [28]. An example of an SPL, given by Rosenmüller et al. [25], is mail client software which relies on a common mail framework. Variations of the mail client software, to support different protocols, for example, would all rely on the same common mail framework [25].

In large enterprise software companies, it is becoming more commonplace to see multiple SPLs, which by comparison, are composed of many interconnected subsystem versions and variants [4]. Multiple SPLs commonly refers to vertically tiered software stacks, with application SPLs and infrastructure SPLs [27], but can also refer to distributed SPLs, as in the case of sensor software [25]. An example of vertically tiered multiple SPLs would be individual application software product lines that all rely on the same database platform, which is itself a software product line [27] [4].

More recently, collaborative partnerships between software product teams and companies are becoming more commonplace, in order to address a larger variety of market segments [6] [11]. These collaborative environments are termed Software Ecosystems (SECOs), and while profitable to the partners involved, they introduce complexity to the management of requirements [14] [13]. A SECO involves a group of software products which rely on a particular technological base.

To illustrate how a SECO works, we can consider a database software product, which provides a technological basis for several application software products.

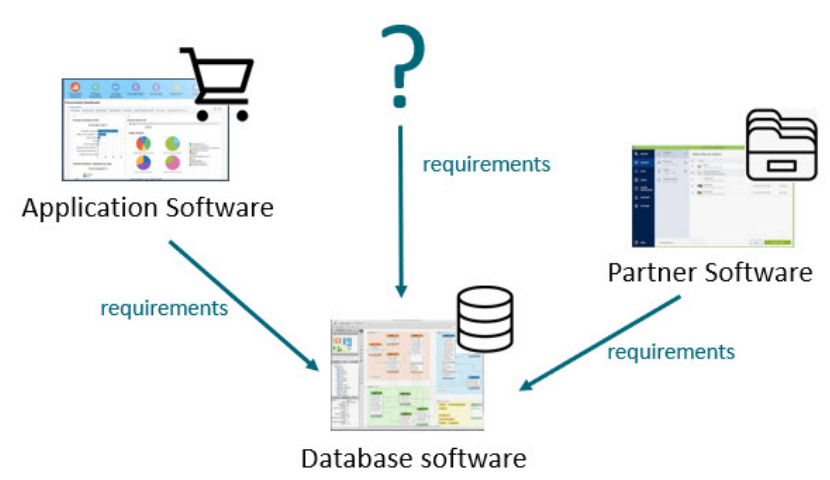

Figure 1: Example of a Software Ecosystem (SECO). 
In this example, there is a procurement software product for managing purchases and invoices. It saves purchase data to the database tables of the database product. Additionally, in the SECO, there is third-party backup archival software, which offers more sophisticated backup capabilities than the database software offers on its own. An enterprise software customer may initially require only the procurement software but ends up also buying the database and backup archival software within the SECO product offerings because they are all well-integrated, and functionally optimized to work together. There is complexity in achieving the integration, which is a main selling point for the customer, but there is a collective profitability to the ecosystem partnership when the customer buys multiple integrated products.

All of these environments - SPLs, multiple SPLs and SECOs add complexity to the process of requirements collection and management. This research takes a focus on one aspect of requirements management, the identification of stakeholders, as a preliminary step of requirements elicitation, and isolates the topic to the SECO environment. The aim is to create a foundation of high-quality research for the optimization of stakeholder identification that then could be extended to SPL environments, or act as a foundation for further requirements management research within the SECO environment.

In the given SECO example, the identification of the actors within the SECO is straightforward. We can imagine that the database development team knows of the application software products, however, in the real world, this is not always the case. Many platform software products (databases, operating systems, application platforms) have open interfaces [30], which allow any software within the same network to interface with them, without notifying the platform software product team of the integration [15] [12]. Open interfaces are desirable, as they allow for easier extensibility and integration [30]. Likewise, the number of stakeholders may become too large to manage completely [9]. This presents challenges in the case of requirements elicitation.

For any software product, the first step of requirements management is the elicitation of requirements [21] [5], but before this can occur, it is imperative to know from whom the requirements should be elicited [15] [26]. The person (or company) making requirements to software products are called 'stakeholders', and without identifying a representative group of stakeholders before the requirements elicitation process, the software is likely developed with an incomplete view of the stakeholder needs, which in turn leads to poor adoption or a delayed timeline to compensate for late requirements [1] [23]. These factors are both very expensive, and harmful to the reputation of the software company [9] [1] [19]. Furthermore, requirements elicitation and stakeholder identification is iterative and repetitive process [15] [2].

The relationships between SECO members are the foundation of the success of the SECO [15] [14]. Therefore, for SECOs, the process of identifying stakeholders is even more critical than for traditional standalone software products because the success of the SECO depends on the integration of products and services of actors in the ecosystem. This research refers to 'key' stakeholders as the stakeholders which are critical for requirements elicitation process, and the exact definition of what makes a key stakeholder varies depending on the software project. Typically, key stakeholders have influence, power, represent other stakeholders, or target a niche market and/or region that is strategic for the SECO. In essence, a key stakeholder is likely someone from whom a late requirement could derail the initial software planning, and disrupt the development timeline.

These issues can be addressed with a reference process model, which can be applied by requirements managers of the technological base software of a SECO, in order to design a business process model for stakeholder identification. The business process model should make use of the stakeholder network to identify attributes (or characteristics) of the stakeholders, which support the decision of who in the SECO are the key stakeholders, from whom requirements must be elicited.

The resulting contribution of the research will be a reference process model, which has been designed with the support of literature sources and practitioner collaboration, evaluated in a controlled (artificial) environment, and ultimately evaluated in a naturalistic environment: design-inherent (ex-ante, focus group study) and outcome-related (ex-post, web-based survey).

\section{RESEARCH QUESTIONS}

The objective of the current research is to investigate stakeholder identification processes as part of requirements elicitation to ensure that a sufficient process for stakeholder identification in SECOs exists. Many of the existing processes for stakeholder identification were designed before SECOs became prominent, and therefore do not account for the complexity of the stakeholder landscape and relationships.

Software ecosystems typically have many types of stakeholders, some which may be integrating with the ecosystem using open interfaces and are thus unknown [15] [12]. Therefore, the attributes and roles of stakeholders in the ecosystem should be exploited to get the most comprehensive view of the stakeholder network in the ecosystem.

\subsection{Hypothesis}

Based on the research objective, the following hypothesis can be made:

It is not necessary to identify every stakeholder in a SECO to achieve comprehensive requirements elicitation, as long as key stakeholders are identified.

\subsection{Research Questions}

The following research questions must be answered in order to confirm or deny the hypothesis:

2.2.1 Main Research Question. How can key stakeholders be identified as part of the requirements elicitation process for SECOs?

\subsubsection{Sub Research Questions.}

- (RQ1) Which stakeholder identification processes exist in literature and in practice, and which ones can be adapted for SECOs?

- (RQ2) Which stakeholder attributes are most often referred to in literature and used by practitioners, and which are minimally required to define SECO stakeholders? 
- (RQ3) How can a valid reference process model for stakeholder identification in SECOs be designed?

\section{RESEARCH METHODOLOGY AND APPROACH}

The Design Science Research Methodology (DSRM) is one of the most distinctive approaches for Information Systems (IS) research projects, because of the guidance it provides in iterative design improvements and evaluation [8] [24]. DSRM positions itself as a problem-solving paradigm [24] with the objective of producing an artifact that has been designed and then evaluated thoroughly [22] [7]. It is technology-oriented and its products are assessed against criteria of value and utility [17]. DSRM is focused on creations of artificial systems, and it addresses the building and evaluation of artifacts to meet business needs [22]. So-called "wicked problems" problems that exist in vicious circles, or lack self-evident solutions - are good candidates for a DSRM approach [8].

The objective of this research is to examine how key stakeholders can be identified in complex SECOs. The main component of the current research is the design of an artifact in the form of a reference process model, that is applied in a real-world environment to develop a business process model. The task of identifying all stakeholders in a SECO is often not possible due to the high volume of stakeholders and open interfaces. There is no self-evident solution for how to identify the necessary stakeholders. Furthermore, when stakeholders are not adequately identified, it translates to an impact on the development project. This indicates that there is a business need for an artifact (process) that is built and evaluated in a real-world environment. Therefore, DSRM provides an appropriate approach for this research.

More specifically, this research follows the DSRM approach originally proposed by Peffers [24] and extended by Ostrowski [22], who offers methods for the meta-design phase and provides, in particular, a detailed approach for the design of a reference process model artifact.

Figure 2 depicts the current research scope mapped to the DSRM proposed by Ostrowski [22].

\subsection{Identifying the Problem, Motivation, and Research Objectives}

The problem has been identified by performing literature reviews. The outcome of this stage of the research was a selection of literature of stakeholder identification processes, and literature referencing stakeholder attributes.

\subsection{Designing \& Developing and Demonstration}

Two early artifacts were designed, a categorization of stakeholder identification process steps and a stakeholder attribute taxonomy, using literature and practitioner focus groups to shape the design. These early artifacts contribute to the design of the main artifact the reference process model - which is generic in nature. The very early design of the generic reference process model, used to convey an idea of the end artifact, can be seen in Figure 6. It will then be evaluated artificially in a controlled environment, and changes will be made to the model as necessary in the design phase.

\subsection{Evaluation and Communication}

Evaluation proves that a research artifact achieved its purpose. A design science research artifact is assessed against the criteria of value and utility [17]. The results will be communicated in journals, at conferences, and as part of a $\mathrm{PhD}$ thesis.

The reference process model will be used in a naturalistic environment as part of a focus group study (ex-ante) and validated with a web-based survey (ex-post) to evaluate semantic and pragmatic qualities, with a syntactic evaluation of the BPMN notation. Ideally, the reference process model would be evaluated in case studies over the period of several requirements elicitation cycles. However, this would take years to perform, and there is not enough time within the scope of a $\mathrm{PhD}$ to perform that type of long-term evaluation. While the plan to evaluate the artifact using focus groups and a web-based survey follows rigorous design science practices, it is possible that the evaluation is somewhat limited compared to other long-term or in-depth approaches.

\section{PRELIMINARY RESULTS}

The results in this section are early results addressing the literature review and synthesis, RQ1 and RQ2, and the expected outcome of the research.

\subsection{Literature Review}

As a requirements manager in an enterprise software company, I have experienced and observed first-hand the challenges that practitioners face with regards to requirements management in complex software environments.

Initial scoping reviews to this topic area revealed that SECO research was still developing. Manikas et al. performed the first systematic literature review on SECOs in 2013 [16], stating that research in SECOs is still in its infancy. This statement was confirmed by my own lightweight empirical results from plotting the cumulative Google Scholar results on the search term "software ecosystem" for every completed year from the first mention (1968) until today.

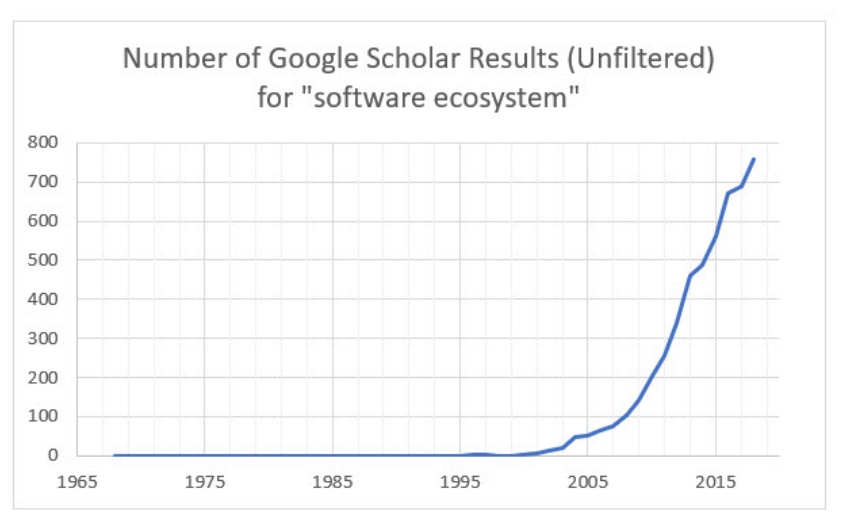

Figure 3: Accumulated SECO literature. 


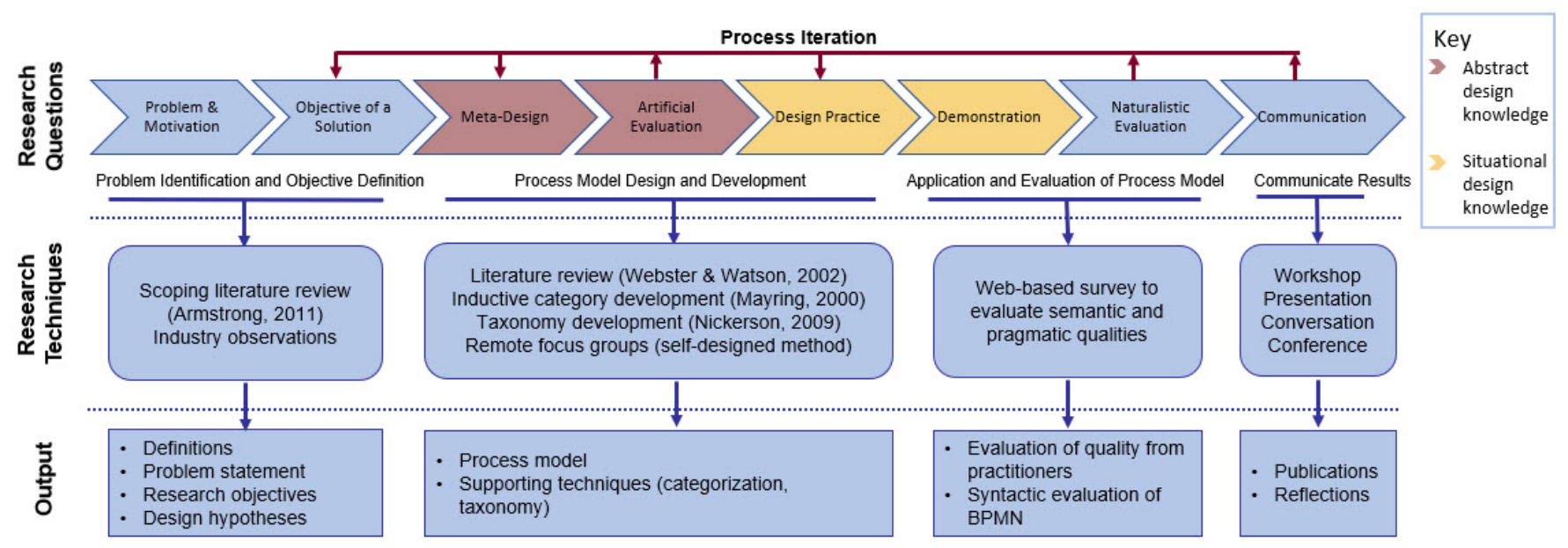

Figure 2: Design science methodology (DSRM) applied to research.

Since around 2010, the number of SECO publications steadily increases every year and continues to trend upwards. This indicates that academic contributions to the domain of SECO research are still very much relevant.

Subsequently, it was necessary to determine which focus area would be appropriate within the domain of requirements engineering. Although practitioners mentioned requirements prioritization as being of particular importance, the scoping review revealed that the prerequisite phase to requirements prioritization, requirements elicitation, was not documented in the context of SECOs. Therefore, literature to requirements elicitation was reviewed, and each step was scoped individually. Indeed, even the initial step within requirements elicitation, identifying stakeholders, was underdeveloped, and therefore it was chosen to be the focus of the research.

The initial systematic literature review (SLR1) targeted stakeholder identification in SECOs, but only returned 19 unique results, none of which presented a prescriptive approach. Therefore, second and third systematic literature reviews were performed. The second systematic literature review (SLR2) explored stakeholder identification processes within the context of requirements elicitation and did not limit the product line environment. The third systematic literature review (SLR3) discovered stakeholder attributes (or characteristics) in SECOs. The basis of identification is being able to define something or someone by a fixed set of parameters. Stakeholder attributes are particularly vital in the context of SECOs where the strength and success of complex relationships provide the foundation of the SECO health [10] [14] [3].

4.1.1 Literature Review and Analysis Process. Based on the methodology from Webster and Watson [31], multiple systematic literature reviews were conducted to obtain a sound scientific basis of SECO concepts, stakeholder attributes and stakeholder identification processes. In the context of this research agenda, the literature review establishes a basis for later collecting and analyzing practitioner insight to the stakeholder identification steps and commonly tracked stakeholder attributes as applicable to SECO environments.

Four databases (ScienceDirect, Scopus, SpringerLink, and Web of Science) were used to find high-quality content.
Table 1: Systematic literature review results.

\begin{tabular}{llllll}
\hline Step & Selection Criteria & $\begin{array}{l}\text { SLR1 } \\
\text { Results }\end{array}$ & $\begin{array}{l}\text { SLR2 } \\
\text { Results }\end{array}$ & $\begin{array}{l}\text { SLR3 } \\
\text { Results }\end{array}$ & $\begin{array}{l}\text { Total } \\
\text { Results }\end{array}$ \\
\hline 1 & $\begin{array}{l}\text { Dedicated } \\
\text { keyword-based } \\
\text { search strings in }\end{array}$ & 18 & 26 & 244 & 288 \\
& journal databases & & & & \\
2 & Full text review & 9 & 10 & 28 & 47 \\
3 & Backward search & - & +10 & +16 & +26 \\
Result: & 9 & 20 & 44 & 73 \\
\hline
\end{tabular}

Table 2: Search strings used in each systematic literature review.

\begin{tabular}{ll}
\hline SLR No. & Corresponding Search String \\
\hline SLR 1 & $\begin{array}{l}\text { software ecosystem AND ("stakeholder identification" } \\
\text { OR "identify* stakeholder" } \\
\text { OR "identify* actor*") }\end{array}$ \\
SLR 2 & $\begin{array}{l}\text { requirement* AND elicitation AND "identification pro- } \\
\text { cess" AND ("stakeholder identification" OR "identify } \\
\text { stakeholder*") }\end{array}$ \\
SLR 3 & $\begin{array}{l}\text { "software ecosystem" AND (stakeholder* OR actor } \\
\left.\text { AND (characteristic* } \text { or attribute }^{*}\right)\end{array}$ \\
\hline
\end{tabular}

The keywords used in this literature review are as follows: software ecosystem, stakeholder/actor identification, requirement elicitation, identification process, stakeholder characteristic/attribute. They were combined in the search strings in Table 2 .

The papers included in this literature review are from peerreviewed high-quality journals and conferences, which can be easily achieved by using databases that only index high-quality content. Papers excluded from this literature review are not in English language, and do not focus on the process of stakeholder (or actor) 


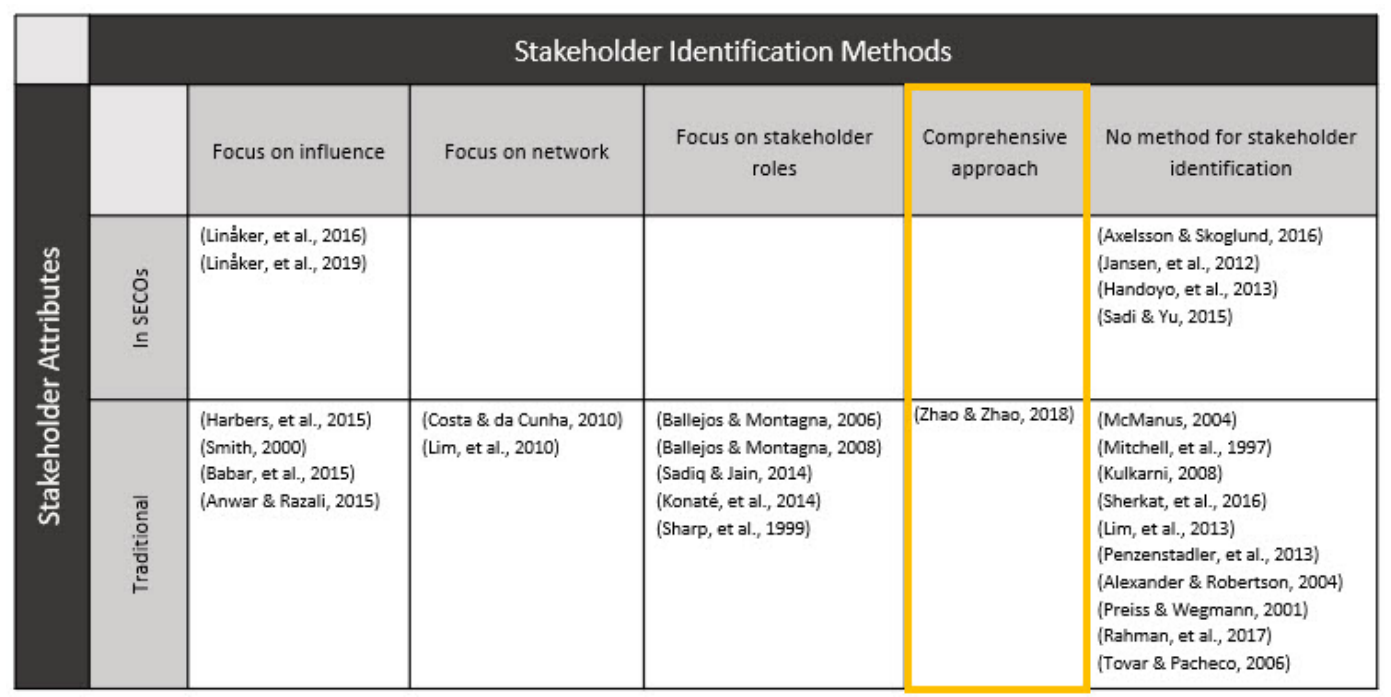

Figure 4: Synthesis of selected literature - concept matrix.

identification in the body of the paper and are not free of charge under the library license of Maynooth University.

\subsubsection{Literature Review Synthesis. The systematic literature review} returned the following results:

- 288 reviewed conference papers and articles in total,

- Reduced to 73 selected sources on which the research is based,

* 28 of the 73 mention stakeholder identification processes and/or attributes

- 15 of the 73 included processes, methods, or frameworks for identifying software stakeholders,

- 20 of the 73 included attributes of software stakeholders.

The 28 papers and articles were synthesized together to identify common themes and research gaps, which are summarized in Figure 4.

The remaining conference papers and articles (73 less 28 synthesized in this section) provide a foundation of literature surrounding SECOs and requirements elicitation. They will provide an anchor in theory in the future of the research project when design decisions need to be made.

Figure 4 represents common concept areas within the selected literature. As SECOs are still an emerging field of research, most of the literature around stakeholder identification is written in the context of a traditional software project, where the dependencies between stakeholders are not as complex.

Typically, in the selected literature, it is more common to see detailed descriptions of stakeholder attributes, which include roles (keystone player, niche players, etc.), participation level, geographical location, and more. In the literature that proposes a stakeholder identification method, there is almost certainly also mention of stakeholder attributes, since many of the process steps involve defining particular attributes of a stakeholder in a given order. This is the motivation for classifying the literature based on both stakeholder identification process and stakeholder attribute components.

It was discovered that most of the literature, where a stakeholder identification process is present, aims to optimize the stakeholder identification process with a particular focus. Linåker et al. [15], for example, focus their research on identifying the stakeholders within the SECO that have a large amount of influence on the decisionmaking over the rest of the ecosystem actors. The other common stakeholder identification focuses are on identifying more stakeholders through known stakeholders and identifying stakeholders of noteworthy roles. Only Zhao et al. [32] propose a stakeholder identification process that addresses, at a high-level, identifying stakeholders with influence, and within certain roles (including the keystone actor) while building the network. This is considered to be a comprehensive approach, because it covers a broader attribute scope.

It is clear that from the analysis of the literature that there is a lack of comprehensive approaches to stakeholder identification, and especially in the context of SECOs. This lack of and need for a comprehensive approach is also echoed by observed practitioners and industry experience. One of the goals of this research project is to make a contribution that is generalized, such that it is optimized for a SECO environment, but it not limited to a particular SECO type. Providing this type of comprehensive approach to stakeholder identification in SECOs would also lay the foundation for further research in requirements elicitation in SECOs.

By performing the concept-based analysis on the selected literature, the knowledge gap has been confirmed, and the sources have been identified, which will be used to generate early artifacts - stakeholder identification process step categories and a taxonomy of stakeholder attributes.

\subsection{Preliminary Results to RQ1}

The first research question "Which stakeholder identification processes exist in literature and in practice, and which ones can be 
adapted for SECOs?" was addressed using a systematic literature review in accordance with the systematic literature review process by Webster and Watson [31] and following the inductive category development proposed by Mayring [18].

To induce the categories depicted in Table 3 , first the criterion of selection was defined. To be considered for a category, the literature must have contributed a novel process, model or framework which had multiple steps detailing how to identify the stakeholder. The analysis returned 58 process steps . Next, the first $50 \%$ of the process set steps were assessed and categories were induced and combined when there was a strong resemblance. A check was performed to ensure the analysis was reliable. Then the rest of the material was worked through, again creating new and combining categories where appropriate. A summative reliability check was performed.

The final result of the inductive category development process is a list of 15 stakeholder identification steps depicted in Table 3 , listed in order of prevalence in the literature. The next step is to collect stakeholder identification process steps in a comprehensive focus group study and compare the results to these initial results.

Table 3: Categories of Steps in Selected Stakeholder Identification Methods.

\begin{tabular}{ll}
\hline No. & Description \\
\hline 1 & Identify initial set of stakeholders \\
2 & Assess influence and power \\
3 & Analyze and rate stakeholders \\
4 & Assess interest and participation \\
5 & Specify all possible stakeholder roles \\
6 & Define project scope \\
7 & Model network \\
8 & Select stakeholders \\
9 & Associate individual stakeholders to roles \\
10 & Build network \\
11 & Specify all possible stakeholder types \\
12 & Elicit requirements \\
13 & Prioritize stakeholders \\
14 & Outline assumptions and risks \\
15 & Repeat \\
\hline
\end{tabular}

\subsection{Preliminary Results to RQ2}

The second research question "Which stakeholder attributes are most often referred to in literature and used by practitioners, and which are minimally required to define SECO stakeholders?" was addressed using a systematic literature review in accordance with the systematic literature review process by [31] and following the taxonomy development proposed by Nickerson [20].

To deduce the taxonomy depicted in Figure 5, first the metacharacteristic for the taxonomy was defined.

For this research, the meta-characteristic is the ability of the attribute to distinguish stakeholders from one another. Then the subset of objects (attributes) derived from the selected literature were examined. Next, 23 distinguishing characteristics were identified, and the characteristics were grouped into four dimensions (type, role, relationship and value). The result is the first taxonomy, which will next be reviewed for additional conceptualizations that might not have been identified in the original empirical data. Once a final taxonomy has been deduced from empirical data in the literature, a focus group study will be performed to collect stakeholder attributes and compare the results to these initial results.

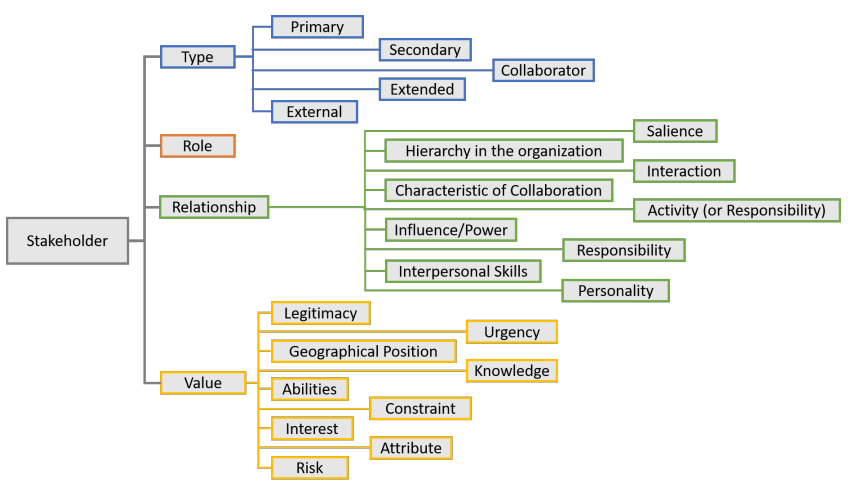

Figure 5: Taxonomy of stakeholder attributes.

\subsection{Expected Outcome}

The expected outcome of the research project is a model artifact, specifically a reference process model, similar to the very early draft depicted in Figure 6, which combines the process steps and stakeholder attributes from the early artifacts. The process model takes the perspective of the requirements manager of the common technological base software (from the example earlier, that would be the database). The requirements manager first identifies stakeholders that they already know of and documents their roles in the SECO. They then open the lines of communication with the known (or primary) stakeholders and request them to identify further (secondary) stakeholders. The requirements manager from the technological base software now has a more expansive view of the stakeholders in the SECO, and can describe the relationships between the stakeholders to identify which stakeholders are, at a minimum, key for a requirements elicitation cycle.

This process model uses BPMN notation, and will go through several iterations of design relying on literature and practitioner input before it is evaluated.

\section{WORK PLAN}

I am a part-time student, therefore my research is spread over a longer period of time than for a traditional PhD student. Until now, the research has focused on literature review, observation of industry practices, and the early artifact design described in previous sections.

In the summer months of 2020, it is planned to develop a methodology for conducting remote focus groups based on an existing technique using Post-It Notes [29] and using Mural digital workspace software. In the proposed method, practitioners use existing process steps and attributes, provided by the early outputs of this research, or write new ones on (virtual) sticky notes, and then order them as an illustration of the investigated process. Based on this methodology, a deductive approach is applied to collect empirical data from 


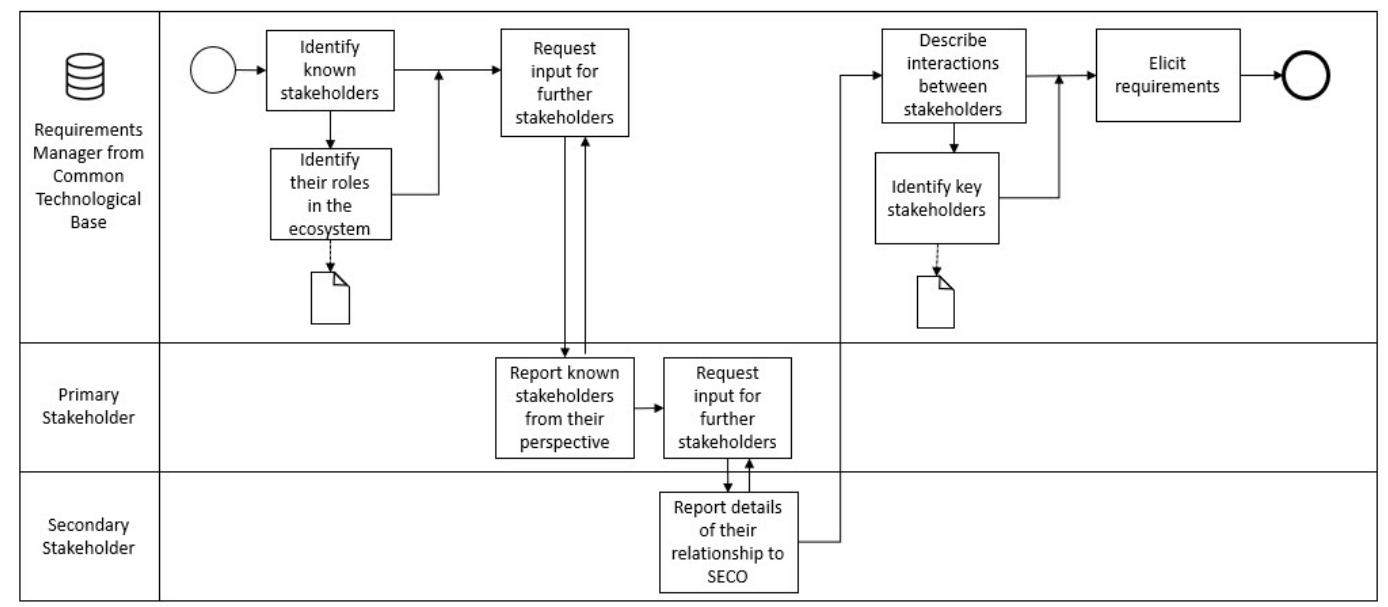

Figure 6: Expected outcome - reference process model.

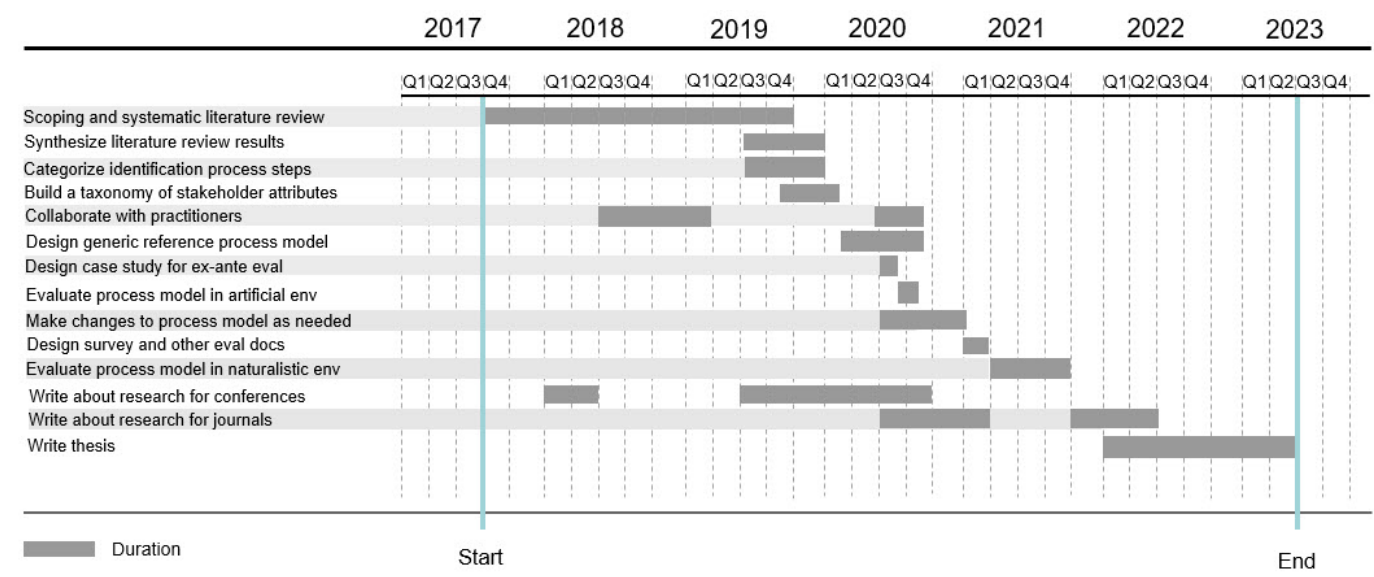

Figure 7: Gantt chart of work plan

practitioners regarding the ideal combination of the stakeholder identification process step categories and stakeholder attributes.

After collecting and synthesizing the empirical results, a journal article will be submitted to the Requirements Engineering Journal, which describes the development of the early outputs (categorization and taxonomy), the focus groups with practitioners, and a synthesis of the results.

A conference paper focusing on the methodological contribution will be prepared for the International Conference on Design Science Research in Information Systems and Technology (DESRIST).

Based on the current understanding of the research problem, the initial collaboration with practitioners and the synthesis of the literature, it has already been possible to create a very early draft of a reference process model (see Figure 6), which is generic in nature. The reference process model will, in parallel to and informed by focus group activities, undergo several design iterations throughout the year as part of the meta-design phase in the design science research methodology. The final part of the meta-design phase is the artificial evaluation of the artifact, which will take place in an artificial environment with controlled variables.

In 2021, the naturalistic design phase will take place, where the reference process model will be evaluated with real users, most likely in a web-based survey to evaluate semantic and pragmatic qualities, with a syntactic evaluation of the BPMN notation. The reference process model will inform the design of instantiations of business process models in real-world environments.

The year of 2022 and the first half of 2023 are reserved for an additional journal contribution based on the case studies and validation of the reference process model, and for writing the thesis and preparing for the viva voce examination.

\section{REFERENCES}

[1] Wasim Alsaqaf, Maya Daneva, and Roel Wieringa. 2019. Quality requirements challenges in the context of large-scale distributed agile: An empirical study. Information and software technology 110 (2019), 39-55.

[2] S Celar, M Turic, and L Vickovic. 2010. Stakeholder Analysis: Process Modell. DAAAM International Scientific Book (2010), 489.

[3] Rebeca T da Silva, F Luiz Gustavo, Edneuci D Audacio, and Elias C Genvigir. 2017. Identifying actors to support software ecosystem health. In 2017 IEEE/ACM 
foint 5th International Workshop on Software Engineering for Systems-of-Systems and 11th Workshop on Distributed Software Development, Software Ecosystems and Systems-of-Systems ( FSOS). IEEE, 76-77.

[4] Ferruccio Damiani, Ina Schaefer, and Tim Winkelmann. 2014. Delta-oriented multi software product lines. In Proceedings of the 18th International Software Product Line Conference-Volume 1. 232-236.

[5] Diego Dermeval, Jéssyka Vilela, Ig Ibert Bittencourt, Jaelson Castro, Seiji Isotani, Patrick Brito, and Alan Silva. 2016. Applications of ontologies in requirements engineering: a systematic review of the literature. Requirements Engineering 21, 4 (2016), 405-437.

[6] Remo Eckert, Matthias Stuermer, and Thomas Myrach. 2019. Alone or Together? Inter-organizational affiliations of open source communities. Fournal of Systems and Software 149 (2019), 250-262.

[7] Markus Helfert, Brian Donnellan, and Lukasz Ostrowski. 2012. The case for design science utility and quality-Evaluation of design science artifact within the sustainable ICT capability maturity framework. Systems, Signs and Actions An International fournal on Information Technology, Action, Communication and Workpractices 6, 1 (2012), 46-66.

[8] Alan R Hevner, Salvatore T March, Jinsoo Park, and Sudha Ram. 2004. Design science in information systems research. MIS quarterly (2004), 75-105.

[9] Fadhl Hujainah, Rohani Binti Abu Bakar, and Mansoor Abdullateef Abdulgabber 2019. StakeQP: A semi-automated stakeholder quantification and prioritisation technique for requirement selection in software system projects. Decision Support Systems 121 (2019), 94-108.

[10] Sami Hyrynsalmi and Matti Mäntymäki. 2018. Is ecosystem health a useful metaphor? towards a research agenda for ecosystem health research. In Conference on e-Business, e-Services and e-Society. Springer, 141-149.

[11] Marco Iansiti and Roy Levien. 2004. The keystone advantage: what the new dynamics of business ecosystems mean for strategy, innovation, and sustainability. Harvard Business Press.

[12] Slinger Jansen, Sjaak Brinkkemper, Jurriaan Souer, and Lutzen Luinenburg. 2012 Shades of gray: Opening up a software producing organization with the open software enterprise model. Journal of Systems and Software 85, 7 (2012), 14951510

[13] Slinger Jansen, Stef Peeters, and Sjaak Brinkkemper. 2013. Software Ecosystems From Software Product Management to Software Platform Management. In IWLCSP@ICSOB. 5-18.

[14] Eric Knauss, Aminah Yussuf, Kelly Blincoe, Daniela Damian, and Alessia Knauss. 2018. Continuous clarification and emergent requirements flows in opencommercial software ecosystems. Requirements Engineering 23, 1 (2018), 97-117.

[15] Johan Linåker, Björn Regnell, and Daniela Damian. 2020. A method for analyzing stakeholders' influence on an open source software ecosystem's requirements engineering process. Requirements Engineering 25, 1 (2020), 115-130.

[16] Konstantinos Manikas and Klaus Marius Hansen. 2013. Software ecosystemsA systematic literature review. Fournal of Systems and Software 86, 5 (2013), 1294-1306.

[17] Salvatore T March and Gerald F Smith. 1995. Design and natural science research on information technology. Decision support systems 15, 4 (1995), 251-266.

[18] Philipp Mayring. 2004. Qualitative content analysis. A companion to qualitative research 1, 2004 (2004), 159-176.

[19] John McManus. 2004. A stakeholder perspective within software engineering projects. In 2004 IEEE International Engineering Management Conference (IEEE Cat. No. 04CH37574), Vol. 2. IEEE, 880-884.

[20] Robert Nickerson, Jan Muntermann, Upkar Varshney, and Henri Isaac. 2009 Taxonomy development in information systems: Developing a taxonomy of mobile applications. (2009).

[21] Ibtehal Noorwali, Nazim H Madhavji, Darlan Arruda, and Remo Ferrari. 2019. Towards a Meta-Model for Requirements-Driven Information for Internal Stakeholders. In International Working Conference on Requirements Engineering: Foundation for Software Quality. Springer, 262-278.

[22] Łukasz Ostrowski. 2014. Design and evaluation of activities and reference model for the meta-design phase of design science-demonstrated on business process model artefacts. Ph.D. Dissertation. Dublin City University.

[23] Carla Pacheco and Ivan Garcia. 2012. A systematic literature review of stakeholder identification methods in requirements elicitation. Fournal of Systems and Software 85, 9 (2012), 2171-2181.

[24] Ken Peffers, Tuure Tuunanen, Marcus A Rothenberger, and Samir Chatterjee. 2007. A design science research methodology for information systems research fournal of management information systems 24, 3 (2007), 45-77.

[25] Marko Rosenmüller, Norbert Siegmund, Christian Kästner, and Syed Saif ur Rahman. 2008. Modeling dependent software product lines. In Proceedings of the GPCE Workshop on Modularization, Composition and Generative Techniques for Product Line Engineering (McGPLE). 13-18.

[26] Mahsa H Sadi and Eric Yu. 2015. Designing software ecosystems: How can modeling techniques help? In Enterprise, Business-Process and Information Systems Modeling. Springer, 360-375.

[27] Horst Schirmeier and Olaf Spinczyk. 2009. Challenges in software product line composition. In 2009 42nd Hawaii International Conference on System Sciences.
IEEE, $1-7$

[28] David Sellier, Mike Mannion, and Jason Xabier Mansell. 2008. Managing requirements inter-dependency for software product line derivation. Requirements engineering 13, 4 (2008), 299-313.

[29] David Straker. 2009. Rapid problem solving with post-it notes. Da Capo Lifelong Books.

[30] Damian A Tamburri, Fabio Palomba, Alexander Serebrenik, and Andy Zaidman. 2019. Discovering community patterns in open-source: a systematic approach and its evaluation. Empirical Software Engineering 24, 3 (2019), 1369-1417.

[31] Jane Webster and Richard T Watson. 2002. Analyzing the past to prepare for the future: Writing a literature review. MIS quarterly (2002), xiii-xxiii.

[32] Junwen Zhao and Yuemin Zhao. 2018. A Systematic Stakeholder Selection Model in Requirements Elicitation for Software Projects: A Systematic Mapping Study. Master's thesis. Blekinge Institute of Technology, Karlskrona, Sweden. 